\title{
Medical Therapy for Secondary Prevention of Atherothrombotic Events in Peripheral Artery Disease
}

\author{
Justin T Morrison, ${ }^{1,2}$ Judith Hsia ${ }^{1,2}$ and Marc P Bonaca ${ }^{1,2}$ \\ 1. Department of Medicine, Division of Cardiovascular Medicine, University of Colorado Anschutz Medical Campus, Aurora, CO, USA \\ 2. Colorado Prevention Center (CPC) Clinical Research, Aurora, CO, USA
}

DOI: https://doi.org/10.17925/HI.2021.15.1.14

$\mathrm{P}$ atients with peripheral artery disease (PAD) are at risk for severe morbidity and mortality, including ischaemic-related events. Furthermore, there is heterogeneity within the PAD population, where the drivers of risk for cardiovascular and limb-specific ischaemic events differ. Patients with PAD with concomitant coronary artery disease are at increased risk for cardiovascular ischaemic events, whereas patients with PAD with a prior history of lower-extremity revascularization are at increased risk for limb-specific ischaemic events. The current therapeutic challenge is identifying these risk factors to tailor therapy optimally for each patient. Additionally, the majority of our current medical therapeutics in patients with PAD have been shown to reduce atherothrombotic events, such as myocardial infarction, stroke and cardiovascular death, with a paucity of medical therapeutics specifically targeting a reduction in limb-specific ischaemic events. Over the past several years, there have been several contemporary clinical trials evaluating antithrombotic agents and their efficacy in reducing limb-specific ischaemic events. Specifically, rivaroxaban, with the addition of aspirin, has emerged as an efficacious therapeutic. In this article, we provide a review of the current clinical burden of PAD, the rationale behind current PAD medical therapeutics and the contemporary trials that have described the benefit of a novel therapeutic in PAD, rivaroxaban.

\section{Keywords}

Peripheral artery disease, acute limb ischaemia, amputation, coronary artery disease, rivaroxaban

Disclosures: Colorado Prevention Center Clinical Research receives grant support from Amgen, AstraZeneca, Bayer, Janone, Janssen, Merck, NovoNordisk, Sanafit and Wraser. Justin T Morrison, Judith Hsia and Marc P Bonaca have no financial or non-financial relationships or activities to declare in relation to this article.

Review process: Double-blind peer review.

Compliance with ethics: This study involves a review of the literature and did not involve any studies with human or animal subjects performed by any of the authors.

Authorship: The named authors meet the International Committee of Medical Journal Editors (ICMJE) criteria for authorship for this manuscript, take responsibility for the integrity of the work as a whole, and have given final approval for the version to be published.

Access: This article is freely accessible at

touchCARDIO.com (C) Touch Medical Media 2021

Received: 29 March 2021

Accepted: 24 June 2021

Published online: 21 July 2021

Citation: Heart International. 2021;15(1):14-9

Corresponding author: Justin Morrison, CPC Clinical Research, 2115 N. Scranton St., Suite 2040, Aurora, CO 80045, USA. E: justin.morrison@cpcmed.org Twitter: @JMorrisonMD

Support: No funding was received in the publication of this article.
Peripheral artery disease (PAD) is a severe manifestation of systemic atherosclerotic disease that commonly refers to lower-extremity arterial involvement. The global burden of PAD is estimated to involve over 200 million patients, with prevalence increasing by $24 \%$ over the past decade. In contrast to myocardial infarction and stroke, which are declining in the USA, the incidence of acute presentations of PAD, such as critical limb ischaemia, is on the rise, leading to an increase in hospitalizations and revascularization procedures. ${ }^{2,3}$ In this review, we will discuss the clinical burden of PAD, the rationale behind current PAD medical therapeutics and recent trials of a novel therapeutic in PAD, rivaroxaban.

\section{Clinical burden of peripheral artery disease}

The spectrum of PAD presentations can range from typical claudication or atypical leg pain to chronic limb-threatening ischaemia, defined as rest pain or tissue loss. The term 'chronic limb-threatening ischaemia' was recommended to replace 'critical limb ischaemia' in 2019 to reflect the continuum of impaired perfusion more clearly. ${ }^{4}$ Although half of patients with PAD do not report typical claudication, functional limitation is present in almost all patients with true flow-limiting PAD. Among those with claudication, 10-20\% will have worsening claudication, with 5-20\% progressing to chronic limb-threatening ischaemia within 5 years. ${ }^{5-7}$ In all-comers with chronic limb-threatening ischaemia, the rates of amputation and mortality at 1 year are $12-32 \%$ and $25-45 \%$, respectively. However, recent data have suggested these rates can be decreased with optimal medical therapy, defined by the Trans-Atlantic Inter-Society Consensus II guidelines, with patients not on optimal medical therapy having an eightfold risk of major amputation and death..$^{9,10}$

Patients with PAD, by nature of diffuse atherosclerosis, are also at increased risk of adverse cardiovascular events. When compared with patients with coronary artery disease (CAD), patients with PAD have an increased risk of myocardial infarction, stroke and cardiovascular death (major adverse cardiovascular events [MACE]), and those with both clinically apparent PAD and CAD have a particularly malignant atherothrombotic phenotype. ${ }^{11,12}$ In a subgroup analysis of the FOURIER trial (Further Cardiovascular Outcomes Research with PCSK9 Inhibition, in Subjects with Elevated Risk; ClinicalTrials.gov identifier: NCT01764633), patients with PAD had a $10.4 \%$ risk of suffering a MACE versus $7.6 \%$ in patients with CAD alone (hazard ratio [HR] 2.07; 95\% confidence interval [CI] $1.42-3.01) .13,14$ 
Although patients with PAD suffer significant morbidity and mortality related to MACE risk, their dominant morbidity is related to major adverse limb events (MALE), that is, amputation and acute limb ischaemia. ${ }^{15}$ This is particularly the case in the $60-70 \%$ of patients with severe PAD and no known CAD. ${ }^{16,17}$ Although the aetiology of amputation may be multifactorial, particularly in patients with diabetes, in those with symptomatic PAD it is largely driven by ischaemia. A more specific and highly feared vascular complication is acute limb ischaemia. The analogous condition to ST elevation myocardial infarction in the limb, acute limb ischaemia is associated with long hospital stays, high rates of amputation, high rates of surgery and intensive care unit stays, and high subsequent mortality. Acute limb ischaemia is a devastating complication of PAD that results from complete vessel occlusion arising from in situ thrombosis, acute graft occlusion or embolism.

Acute limb ischaemia is as frequent as myocardial infarction or stroke in patients with chronic PAD, but almost twofold as common in patients with PAD after lower-extremity revascularization, and is a significant source of severe morbidity and mortality and a burden on the healthcare system. Among patients hospitalized with acute limb ischaemia, duration of hospital stay is a median of 8 days, with $20 \%$ requiring surgery and up to $15 \%$ either dying or unable to return home due to severe functional limitations." In addition, increased rates of acute limb ischaemia are seen in patients who have undergone lower-extremity revascularization. Patients with PAD who have previously undergone revascularization are at a fourfold increase in risk for acute limb ischaemia. ${ }^{18}$ The risk is typically seen early post-procedure; however, this risk remains elevated in the long term, with risk not returning to pre-procedure levels. ${ }^{19}$

Although it is well known that patients with PAD are at risk for both MALE and MACE, heterogeneity exists in the risk profiles predicting cardiovascular and limb-specific adverse events. ${ }^{20}$ Furthermore, most trials that have looked at risk reduction in patients with PAD have been subgroup analyses of patients with CAD. ${ }^{1,21}$ As such, most of our current therapeutics for patients with PAD have targeted risk reduction of atherothrombotic events, including myocardial infarction, stroke and cardiovascular death, through efforts such as lipid management and antithrombotic therapies. However, recent clinical trials have focused on patients with PAD, and in particular, reduction of adverse limb events. These contemporary trials provide the basis for identifying patients at the highest risk of MALE, thereby allowing us to tailor therapy to provide robust risk reduction and decreased incidence of adverse outcomes. ${ }^{16,22}$

\section{Current therapeutics targeting atherothrombotic events in peripheral artery disease}

Antithrombotic therapy, defined for this review as either an antiplatelet or anticoagulant, has been the most widely used medication in the treatment of PAD, along with lipid-lowering. Interestingly, no single antithrombotic agent has been shown to significantly reduce MACE in patients with PAD when compared with placebo in a randomized trial. This highlights the lack of proven medications in PAD. Nonetheless, antithrombotics remain widely used to reduce the atherothrombotic complications of PAD.

\section{Aspirin}

Aspirin is the most widely used antithrombotic agent in patients with PAD, and has a class I designation for its use in patients with PAD in the 2016 American College of Cardiology/American Heart Association (ACC/AHA) guideline document. ${ }^{23}$ The Antithrombotic Trialists' Collaboration performed a meta-analysis evaluating the efficacy of antithrombotic agents in reducing MACE in patients with PAD. ${ }^{24}$ Aspirin was the most widely used agent in the meta-analysis, with antithrombotic agents conferring an overall $22 \%$ odds reduction in cardiovascular events, including myocardial infarction, stroke and vascular death. The collaboration also noted an increase in major extracranial bleeds with antiplatelet therapy (odds ratio 1.6; 95\% Cl 1.4-1.8). ${ }^{19}$ Furthermore, specific doses of aspirin were evaluated where reduced benefit was seen with doses lower than $75 \mathrm{mg}$ and higher than $325 \mathrm{mg}$. As such, the dose of aspirin recommended is between 75 and $325 \mathrm{mg}$ per day in patients with symptomatic PAD. ${ }^{19}$

\section{$P 2 Y_{12}$ inhibitors}

Along with aspirin, $\mathrm{P}_{2} \mathrm{Y}_{12}$ inhibitors have been widely used in patients with $\mathrm{PAD}$, both in secondary prevention and post-intervention. Ticlopidine was the first agent closely examined in the early 1990s. In the Swedish Multicenter Study (STIMS), patients with claudication saw a benefit with ticlopidine versus placebo in reducing MACE; however, ticlopidine did increase rates of reversible leucopenia and thrombocytopenia. ${ }^{25}$ These observations supported the role of antiplatelet monotherapy in patients with symptomatic PAD. Subsequently, clopidogrel was developed and approved for clinical use in 1997 in the USA. Clopidogrel is similar to ticlopidine in that it is an irreversible inhibitor of the $\mathrm{P}_{2} \mathrm{Y}_{12}$ receptor but has fewer side effects, including life-threatening blood dyscrasias. Clopidogrel monotherapy was compared with aspirin monotherapy in the CAPRIE study (Clopidogrel versus Aspirin in Patients at risk of Ischemic Events). ${ }^{26}$ Of note, this trial was completed before many other commonly used secondary preventive therapies currently in use were developed. Overall, clopidogrel was superior to aspirin for MACE prevention, although the relative risk (RR) reduction was modest at $8 \%$. In addition, there was no limb vascular benefit in terms of amputation or other limb outcomes. In a subgroup analysis including patients with $\mathrm{PAD}$, the RR reduction for clopidogrel appeared more robust at $23.8 \%$ (95\% Cl 8.9-36.2). ${ }^{21}$ Although there was benefit seen with clopidogrel in the CAPRIE trial, the absolute benefit for MACE reduction with clopidogrel monotherapy over aspirin in patients with PAD treated with current standard of care is unknown, and there is no established benefit for limb-specific ischaemic events.

The question of whether more potent platelet inhibition monotherapy was beneficial in patients with PAD was further complicated by the unexpected results of the EUCLID trial (Examining Use of Ticagrelor in Peripheral Artery Disease; ClinicalTrials.gov identifier: NCT01732822). ${ }^{27}$ This large trial in a broad population with chronic PAD compared clopidogrel to ticagrelor, a $\mathrm{P}_{2} \mathrm{Y}_{12}$ inhibitor not requiring metabolism to its active form. This trial was initiated after the same comparison showed ticagrelor to be superior for MACE and mortality reduction in patients with acute coronary syndromes. In contrast, the EUCLID population only had $\sim 30 \%$ with concomitant coronary disease and was, therefore, a distinct population. The results, surprisingly, and in contrast to those in acute coronary syndromes, showed that ticagrelor was not superior to clopidogrel in preventing the primary outcome of MACE (HR 1.02; 95\% $\mathrm{Cl} 0.92-1.13)$ or the secondary outcome of acute limb ischaemia (HR 1.03; 95\% Cl 0.79-1.33). ${ }^{17}$ Although this was a neutral trial, it was able to provide invaluable insight into defining risk of MACE and MALE in patients with PAD. Patients with PAD with concomitant CAD had an increased risk of MACE compared with those with PAD alone (HR 1.65; $95 \% \mathrm{Cl} 1.43-1.91)^{28}$

The use of dual antiplatelet therapy (DAPT) has also been evaluated in patients with symptomatic PAD in a subgroup analysis of the CHARISMA trial (Clopidogrel for High Atherothrombotic Risk and Ischemic Stabilization, Management, Avoidance; ClinicalTrials.gov identifier: 
NCT00050817). ${ }^{29}$ The combination of clopidogrel and aspirin versus aspirin alone significantly decreased MACE in the subgroup analysis that included patients with previous myocardial infarction, ischaemic stroke or PAD (HR 0.83; 95\% Cl 0.72-0.96). ${ }^{30}$ However, the combination did not significantly decrease the risk of MACE (HR 0.85; 95\% Cl 0.66-1.08) in a post-hoc analysis that included patients with PAD only, but did increase moderate bleeding (HR 1.36; 95\% Cl 1.03-1.79). ${ }^{31}$

In the PEGASUS-TIMI 54 trial (Prevention of Cardiovascular Events in Patients with Prior Heart Attack Using Ticagrelor Compared to Placebo on a Background of Aspirin-Thrombolysis in Myocardial Infarction 54; ClinicalTrials.gov identifier: NCT01225562) PAD subgroup analysis, the combination of ticagrelor $60 \mathrm{mg}$ and aspirin versus aspirin alone provided a reduction of both MACE (HR 0.69; 95\% Cl 0.47-0.99) and MALE (HR 0.65; $95 \% \mathrm{Cl} 0.44-0.95)$ risk in patients with concomitant PAD and CAD. ${ }^{11,32}$ It is important to note that MALE in PEGASUS-TIMI 54 was a composite of acute limb ischaemia and peripheral revascularization, and did not include amputation. There was a consistent non-significant increased risk of major bleeding with ticagrelor. ${ }^{11}$

These results were also seen in the THEMIS trial (Effect of Ticagrelor on Health Outcomes in Diabetes Mellitus Patients Interventions; ClinicalTrials.gov identifier: NCT01991795). ${ }^{33}$ Only 9\% of patients with stable CAD without previous myocardial infarction or stroke and diabetes mellitus had concomitant PAD, but there was a significant reduction of MALE with the use of ticagrelor and aspirin versus aspirin alone (HR 0.45; $95 \% \mathrm{Cl} 1.82-2.94)$; however, there was a significant increase in major bleeding in the group receiving DAPT (HR 2.32; 95\% Cl 1.82-2.94). ${ }^{34}$ The combination of clopidogrel plus aspirin versus aspirin alone in PAD following surgical revascularization was studied in the CASPAR trial (Clopidogrel and Acetylsalicylic Acid in Bypass Surgery for Peripheral Arterial disease; ClinicalTrials.gov identifier: NCT00174759).35.36 There was no difference between the groups in reduction of the composite outcome of graft occlusion, revascularization, amputation or death (HR $0.98 ; 95 \% \mathrm{Cl} 0.78-1.23)$, but there was an increase in moderate or severe bleeding (HR 2.84; 95\% Cl 1.32-6.08).

\section{Dual antiplatelet therapy}

There are no large, randomized trials evaluating the efficacy of DAPT versus aspirin monotherapy in patients who are post-revascularization. Significant heterogeneity exists between small trials evaluating the efficacy of DAPT in patients post revascularization. ${ }^{37}$ However, there have been a few studies that have suggested a benefit of DAPT in this population. In the MIRROR study (Management of peripheral arterial interventions with mono or DAPT), patients were randomized to DAPT with clopidogrel and aspirin versus aspirin and placebo 6 months prior and 6 months post-revascularization, with balloon angioplasty in the femoropopliteal segments. ${ }^{38}$ After 6 months, clopidogrel was dropped in the treatment arm and all patients received aspirin monotherapy. The 6-month data demonstrated a decrease in target lesion revascularization with the use of DAPT ( $5 \%$ versus $20 \%$, $p=0.04$ ). However, a follow-up study at 12 months demonstrated that the benefit of DAPT no longer persisted when clopidogrel was discontinued (25\% versus $32.4 \%, p=0.35)^{39}$

In the CASPAR trial, the prespecified subgroup of prosthetic grafts did see a significant benefit in the composite primary outcome with DAPT (HR 0.65; 95\% Cl 0.45-0.95). ${ }^{35}$ Due to the dearth of data, specific guideline recommendations vary between the professional societies. The European Society of Cardiology (ESC) provides a lla recommendation for DAPT for at least 1 month after infra-inguinal stent implantation and a IIb recommendation for DAPT following bypass grafting below the knee. ${ }^{40}$ In contrast, the ACC/AHA provide a IIb recommendation for DAPT following lower-extremity revascularization, but do not comment on length of therapy. ${ }^{23}$ There is no consensus on length of therapy, although a survey by the Peripheral Vascular surgery Society revealed the most common duration of therapy was 1-3 months. ${ }^{41}$ Furthermore, several device trials of femoropopliteal stents recommend clopidogrel for 1-3 months and aspirin indefinitely.42-44 There is also no standardized duration of DAPT following drug-eluting stent implantation, with current practices extrapolated from the coronary literature. ${ }^{45}$ This highlights a clear need for randomized, controlled trials to establish a benefit of DAPT following revascularization, as well as duration of treatment.

\section{Vorapaxar}

Strategies testing the combination of an antiplatelet and targeted agents antagonizing the platelet receptor for thrombin have been effective. Vorapaxar, the first-in-class reversible antagonist of protease activated receptor-1, is a potent inhibitor of thrombin-mediated cellular processes. In the TRA2 ${ }^{\circ} \mathrm{P}-\mathrm{TIMI} 50$ trial (Thrombin Receptor Antagonist in Secondary Prevention of Atherothrombotic Ischemic Events-Thrombolysis in Myocardial Infarction 50; ClinicalTrials.gov identifier: NCT00526474) of patients with prior myocardial infarction, stroke or PAD, vorapaxar was found to significantly reduce the risk of MACE (HR 0.87; 95\% 0.80-0.94) balanced by an increase in bleeding (HR 1.66; 95\% Cl 1.43-1.93). ${ }^{46,47}$ In a subsequent analysis of patients with PAD in TRA2 ${ }^{\circ} \mathrm{P}-\mathrm{TIMI} 50$, acute limb ischaemia was decreased by $41 \%$ (HR 0.59; 95\% Cl 0.38-0.93). ${ }^{21}$ Within the PAD population, there was overall risk reduction of MACE (HR 0.85; 95\% Cl 0.73-0.99) and MALE (HR 0.70; $95 \% \mathrm{Cl} 0.53-0.92$ ) with the use of vorapaxar. ${ }^{20}$ However, the analysis also highlighted that the MACE and MALE benefit conferred by vorapaxar was greatest in patients with concomitant CAD and prior revascularization, respectively. This, again, highlights the heterogeneity within the PAD population and the importance of identifying the appropriate patients for therapy.

\section{Vitamin $\mathrm{K}$ antagonists}

The strategy of therapeutic warfarin in combination with aspirin was not effective for improving outcomes in patients with PAD. In the DUTCH BOA trial (Dutch Bypass Oral Anticoagulants or Aspirin), patients with chronic limb-threatening ischaemia undergoing surgical revascularization were randomized to a vitamin $\mathrm{K}$ antagonist plus aspirin versus aspirin alone. ${ }^{48}$ There was no difference between groups in the rate of graft occlusion (HR 0.95; 95\% Cl 0.82-1.11), with a significant increase in major bleeding (HR 1.96; $95 \% \mathrm{Cl} 1.42-2.71$ ). In the WAVE trial (Warfarin Antiplatelet Vascular Evaluation; ClinicalTrials.gov identifier: NCT00125671), patients with symptomatic PAD received an anticoagulant (warfarin or acenocoumarol) and antiplatelet (aspirin, ticlopidine or clopidogrel) versus antiplatelet alone. ${ }^{49}$ The addition of an anticoagulant did not reduce MACE (RR 0.92; 95\% $\mathrm{Cl}$ 0.73-1.16), but was associated with increased life-threatening and moderate bleeding (RR 3.21; 95\% Cl 2.02-5.08). ${ }^{50}$

\section{Rivaroxaban}

The clinical trials discussed thus far have demonstrated reductions in MACE risk in PAD subgroups of trials primarily investigating $C A D$ populations. As such, the 2016 ACC/AHA guideline document for lower-extremity PAD only provides recommendations for antiplatelet use in reducing MACE risk, without any specific recommendations to reduce MALE. ${ }^{23}$ Agents that have been shown to reduce MALE, such as vorapaxar, have received lower-level recommendations. Furthermore, the 2017 ESC PAD guidelines recommend antiplatelet therapy in symptomatic PAD, but leave the choice of agent to the treating providers. ${ }^{40}$ 


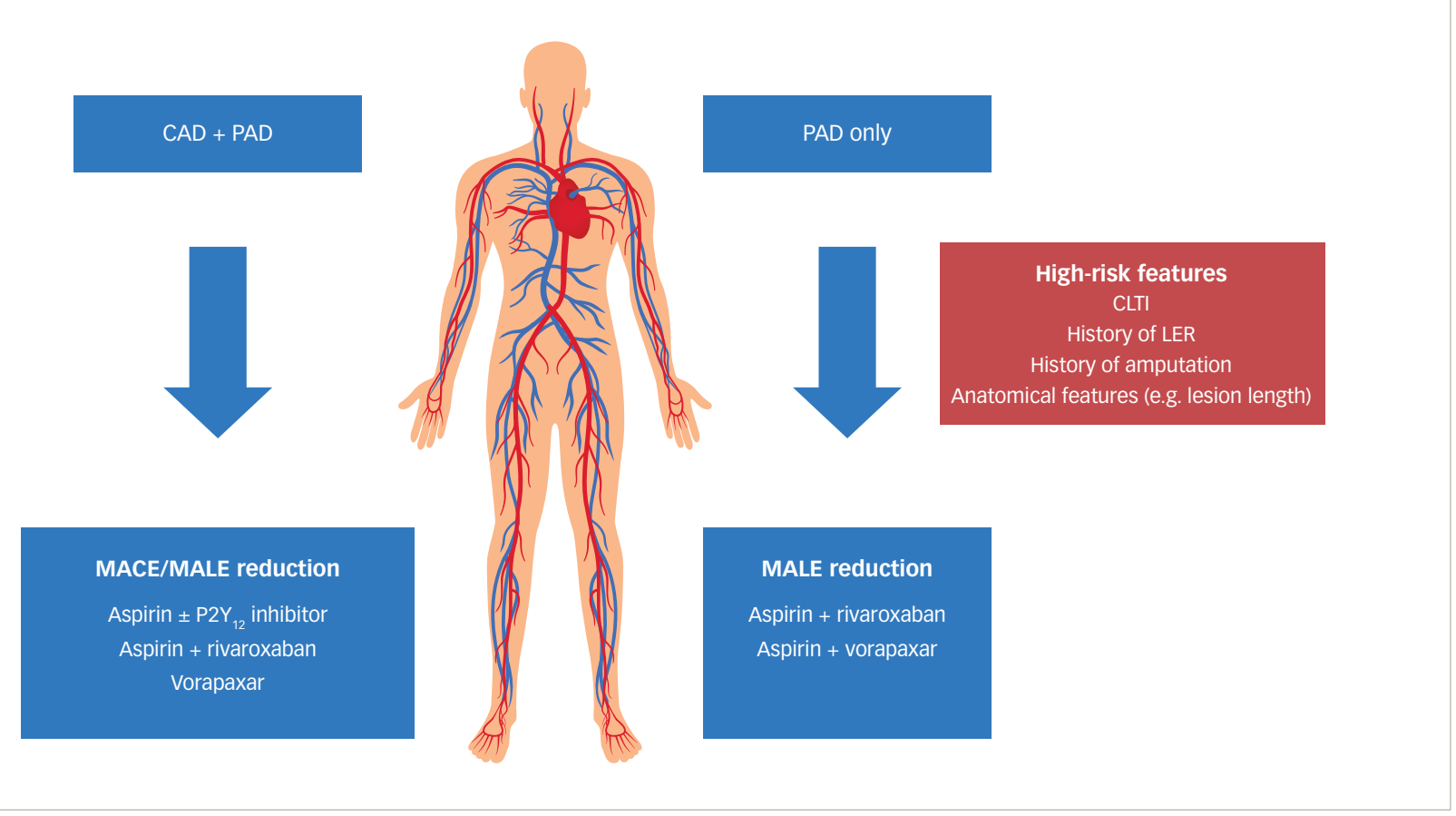

Shown are the recommended medical therapeutics depending on clinical history with high-risk features described in peripheral artery disease patients. $C A D=$ coronary artery disease; $C L T I=$ chronic limb-threatening ischaemia; $L E R=$ lower-extremity revascularization; MACE = major adverse cardiovasCular events; $M A L E=$ major adverse limb events; PAD = peripheral artery disease.

Although subgroup analyses have described MALE reduction with the use of antithrombotic medications, there is a dearth of trials looking at primary PAD populations. Furthermore, these trials have suggested different patients within the PAD population may have different benefits depending on their histories (i.e. concomitant CAD or history of lower-extremity revascularization). The data from vorapaxar suggested that inhibition of thrombin-mediated pathways can modify risk of limb-specific events, specifically acute limb ischaemia. Additional precedent for modulation of this pathway derives from the ATLAS-2 trial (Acute Coronary Syndrome-Thrombolysis in Myocardial Infarction 51; ClinicalTrials.gov identifier: NCT00809965)..$^{51}$ In patients presenting with acute coronary syndromes, low-dose rivaroxaban $2.5 \mathrm{mg}$ twice daily, in addition to DAPT, reduced the risk of MACE (HR 0.84; 95\% Cl 0.72-0.97) with an associated significant increase in thrombolysis in myocardial infarction (TIMI) major bleeding. ${ }^{52}$

The COMPASS trial (Cardiovascular Outcomes for People Using Anticoagulation Strategies; ClinicalTrials.gov identifier: NCT01776424) evaluated the efficacy of low-dose rivaroxaban $2.5 \mathrm{mg}$ with aspirin, rivaroxaban $5 \mathrm{mg}$ alone, and aspirin alone in lowering MACE risk in 27,395 patients with CAD, PAD or both.53,54 The trial demonstrated the combination of low-dose rivaroxaban and aspirin significantly lowered the risk of MACE versus aspirin alone (HR 0.76; 95\% Cl 0.66-0.86), with no significant difference between rivaroxaban alone and aspirin alone. Furthermore, the composite secondary outcomes, including acute limb ischaemia, also demonstrated a marked benefit with the addition of low-dose rivaroxaban to aspirin. These cardiovascular and limb benefits were countered against an increased risk of major and minor bleeding (HR 1.70; 95\% Cl 1.40-2.05). These results included a broad population of patients with CAD and PAD, with approximately $27 \%$ of patients within the study having lower extremity or carotid atherosclerotic disease (PAD). Of these patients with $\mathrm{PAD}$, approximately $56 \%$ were considered to have symptomatic lower-extremity PAD and $66 \%$ had concomitant CAD..$^{55}$
In a subgroup analysis of patients with PAD, the risk of MACE was significantly reduced by $28 \%(\mathrm{HR} 0.72 ; 95 \% \mathrm{Cl} 0.57-0.90)$ with the addition of low-dose rivaroxaban to aspirin. Furthermore, prespecified limb outcomes were evaluated in this population, with a significant decrease in incidence of acute limb ischaemia (HR 0.56; 95\% Cl 0.32-0.99) and major amputations (HR 0.30; 95\% $\mathrm{Cl} 0.11-0.80$ ). The MALE reduction was maintained in the overall PAD, symptomatic PAD and lower-extremity PAD subgroups. There was an increase in major bleeding within this population ( $\mathrm{HR} 1.61 ; 95 \% \mathrm{Cl} 1.12-2.31) .{ }^{55} \mathrm{As}$ such, this was the first trial to demonstrate a reduction in both MACE and MALE in patients with stable $P A D$ treated with low-dose rivaroxaban. Subsequently, the data from this trial were used to secure an additional indication from both the US Food and Drug Administration (FDA) and the European Commission for the combination of rivaroxaban $2.5 \mathrm{mg}$ twice daily and aspirin to reduce the risk of MACE in both patients with CAD and those with PAD. ${ }^{56,57}$

The VOYAGER PAD trial (Vascular Outcomes Study of Acetylsalicylic Acid Along with Rivaroxaban in Endovascular or Surgical Revascularization for PAD; ClinicalTrials.gov identifier: NCT02504216) was a phase III study investigating the efficacy of low-dose rivaroxaban with aspirin versus aspirin alone in patients with PAD who have undergone lower-extremity revascularization. ${ }^{58,59}$ The design of this study was remarkable for several reasons. This investigation was designed to include patients with symptomatic PAD with recent peripheral revascularization as the primary study population. EUCLID, the only prior large outcomes trial in patients with PAD, had included a heterogeneous population. Moreover, the VOYAGER PAD primary composite endpoint was novel, including adjudicated limbspecific outcomes in addition to MACE, a first for a study of this scale.

The results of VOYAGER PAD demonstrated a significant reduction in the composite primary efficacy outcome, which included acute limb ischaemia, major amputation for vascular causes, myocardial infarction, ischaemic stroke and cardiovascular death, of $15 \%$ at 3 years (HR 0.85; 
$95 \% \mathrm{Cl} 0.76-0.96)$. Furthermore, there was a robust reduction in the rate of acute limb ischaemia of $32 \%$ in patients on low-dose rivaroxaban ( $\mathrm{HR}$ 0.67; 95\% Cl 0.55-0.82). Looking at the Kaplan-Meier curves, the separation between the two treatment groups begins to manifest at 3 months, suggesting an early benefit in the primary efficacy outcome with the presence of low-dose rivaroxaban. Between the two study groups, there was an increase in TIMI major bleeding in the study group receiving low-dose rivaroxaban (HR 1.43; 95\% Cl 0.97-2.10). The secondary safety outcome of International Society on Thrombosis and Haemostasis (ISTH)

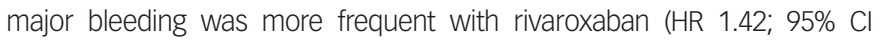
1.10-1.84). There was no increase in the risk of intracranial haemorrhage or fatal bleeding. The authors of the study estimated that the treatment of 10,000 patients with low-dose rivaroxaban in addition to aspirin for 1 year would prevent 181 primary efficacy outcome events at the expense of 29 principal safety outcome events. The results of this study are particularly robust, as approximately $35 \%$ of revascularization procedures were surgical.

Previous studies evaluating antithrombotic therapy in patients post-operative surgical bypass have not demonstrated efficacy and have shown increased bleeding. ${ }^{35,48}$ Furthermore, the reduction in the primary efficacy outcome with the addition of low-dose rivaroxaban was consistent across prespecified subgroups. Taken in totality, VOYAGER PAD demonstrated that the addition of low-dose rivaroxaban provides a robust reduction in MALE in patients with symptomatic PAD who have undergone lower-extremity revascularization. This again highlights the importance of targeting the appropriate population for treatment. When taken together with COMPASS, these two trials strongly support the use of rivaroxaban in patients who have undergone lower-extremity revascularization, especially if there is concomitant CAD (Figure 1). Indeed, based on these data, a supplemental New Drug Application has been submitted to the FDA to expand the indication for rivaroxaban in reducing myocardial infarction, stroke and amputation in patients with PAD following lower-extremity revascularization.
A potential clinical concern that has arisen since the results of VOYAGER PAD were published is the addition of low-dose rivaroxaban in patients on DAPT. In VOYAGER PAD, the addition of clopidogrel was allowed in the protocol, and its use was at the discretion of the treating provider. Approximately $50 \%$ of patients in the study were on clopidogrel at the time of randomization. The interaction between clopidogrel and low-dose rivaroxaban has been investigated in a subsequent analysis. ${ }^{60}$ The reduction of cardiovascular and limb-specific outcomes was consistent regardless of the presence of clopidogrel, with an early benefit in reduction of acute limb ischaemia. Regarding bleeding, there was no difference in major bleeding with the presence of clopidogrel within the first 30 days of therapy. However, with the presence of clopidogrel for greater than 30 days, there was an increase in ISTH bleeding (HR 3.20; $95 \% \mathrm{Cl} 1.44-7.13)$. These data suggest that a short course of clopidogrel after a revascularization procedure with the addition of low-dose rivaroxaban is safe, but that continuation of clopidogrel beyond 30 days is not supported.

\section{Conclusion}

Patients with PAD are at an increased risk for both adverse cardiovascular and limb-specific events. The PAD population is heterogeneous, with differing drivers of MACE and MALE. Specifically, patients who have undergone lower-extremity revascularization are at increased MALE risk, whereas those with concomitant CAD are at increased risk of MACE. Trials of antithrombotic agents have demonstrated MACE reduction in patients with atherosclerotic vascular disease, including those with PAD. Recent trials have identified robust reductions, particularly of acute limb ischaemia and major amputation for ischaemic cause, with rivaroxaban on top of aspirin. As such, the consideration of adding low-dose rivaroxaban in patients with PAD who have recently undergone lower-extremity revascularization or with concomitant CAD is strongly supported by contemporary trial data as we continue to optimize therapy in patients with PAD. $\square$
1. Fowkes FG, Aboyans V, Fowkes FJ, et al. Peripheral artery disease: epidemiology and global perspectives. Nat Rev Cardiol. 2017;14:156-70

2. Virani SS, Alonso A, Benjamin EJ, et al. Heart disease and stroke statistics-2020 update: a report from the American Heart statistics-2020 update: a report from the Am

3. Agarwal S, Sud K, Shishehbor MH. Nationwide trends of hospita admission and outcomes among critical limb ischemia patients: admission and outcomes among critical limb ischemia

4. Conte MS, Bradbury AW, Kolh P, et al. Global vascular guidelines on the management of chronic limb-threatening ischemia J Vasc Surg. 2019;58(Suppl. 1):S1-S109.E33.

5. Hirsch AT, Haskal ZJ, Hertzer NR, et al. ACC/AHA 2005 Practice Guidelines for the management of patients with peripheral arterial disease (lower extremity, renal, mesenteric, and abdominal aortic): a collaborative report from the American Association for Vascular Surgery/Society for Vascular Surgery, Society for Cardiovascular Angiography and Interventions, society for Vascular Medicine and Biology, socien of Interventional Radiogy, and the ACC/AHA Task Fociety of inters tonal Radiogy, and the ACC/AHA Task Force on Practice Guidelines (Whing Committee to Develop Guidel of Cardiovascular and Pulmonary Rehabilitation; National Heart, Lung, and Blood Institute; Society for Vascular Nursing TransAtlantic Inter-Society Consensus; and Vascular Disease Foundation. Circulation. 2006;113:e463-654

6. Sigvant $B$, Lundin $F$, Wahlberg $E$. The risk of disease progression in peripheral arterial disease is higher than expected: a meta-analysis of mortality and disease progression in peripheral arterial disease. Eur J Vasc Endovasc Surg. 2016;51:395-403

7. McDermott MM, Greenland P, Liu K, et al. Leg symptoms in peripheral arterial disease: associated clinical characteristics and functional impairment. JAMA. 2001;286:1599-606.

8. Benoit E, O'Donnell TF Jr., Kitsios GD, lafrati MD. Improved amputation-free survival in unreconstructable critical limb ischemia and its implications for clinical trial design and quality measurement. J Vasc Surg. 2012;55:781-9.

9. Chung J, Timaran DA, Modrall JG, et al. Optimal medical therapy predicts amputation-free survival in chronic critical limb ischemia. J Vasc Surg. 2013;58:972-80

10. Norgren L, Hiatt WR, Dormandy JA, et al. Inter-society consensus for the management of peripheral arterial disease
(TASC II). J Vasc Surg. 2007;45(Suppl. S):S5-67.

11. Bonaca MP, Bhatt DL, Storey RF, et al. Ticagrelor for prevention of ischemic events after myocardial infarction in patients with peripheral artery disease J Am Coll Cardiol 2016;67:2719-28.

12. Bonaca MP, Nault P, Giugliano RP, et al. Low-density lipoprotein cholesterol lowering with evolocumab and outcomes in patients with peripheral artery disease: insights from the patients with peripheral artery disease: insights from the
FOURIER trial (further cardiovascular outcomes research with FOURIER trial (further cardiovascular outcomes research with
PCSK9 inhibition in subjects with elevated risk). Circulation. PCSK9 inhibition

2018;137:338-50.
13. Branch KR, Probstfield JL, Eikelboom JW, et al. Rivaroxaban with or without aspirin in patients with heart failure and chronic coronary or peripheral artery disease. Circulation. 2019;140:529-37.

14. ClinicalTrials.gov. Further cardiovascular outcomes research with PCSK9 inhibition in subjects with elevated risk (FOURIER). ClinicalTrials.gov Identifier: NCT02569242. Available at: https://clinicaltrials.gov/ct2/show/NCT01764633 (accessed 12 July 2021).

15. Kumbhani DJ, Steg PG, Cannon CP, et al. Statin therapy and long-term adverse limb outcomes in patients with peripheral artery disease: insights from the REACH registry. Eur Heart J. 2014:35:2864-72.

16. Bonaca MP, Bauersachs RM, Anand SS, et al. Rivaroxaban in peripheral artery disease after revascularization. N Eng/ J Med. 2020;382:1994-2004

17. Hiatt WR, Fowkes FG, Heizer $G$, et al. Ticagrelor versus clopidogrel in symptomatic peripheral artery disease. N Eng/ J Med. 2017;376:32-40.

18. Jones WS, Baumgartner I, Hiatt WR, et al. Ticagrelor compared with clopidogrel in patients with prior lower extremity revascularization for peripheral artery disease. Circulation. 2017;135:241-50

19. Hess CN, Wang TY, Weleski Fu J, et al. Long-term outcomes and associations with major adverse limb events after peripheral artery revascularization. J Am Coll Cardiol. 2020;75:498-508.

20. Qamar A Morrow DA, Creager MA, et al. Effect of vorapaxa on cardiovascular and limb outcomes in patients with peripheral artery disease with and without coronary artery disease: analysis from the TRA $2^{\circ} \mathrm{P}$-TIMl 50 trial. Vasc Med. 2020;25:124-32.

21. Bonaca MP, Gutierrez JA, Creager MA, et al. Acute limb ischemia and outcomes with vorapaxar in patients with peripheral artery disease: results from the trial to assess the effects of vorapaxar in preventing heart attack and stroke in patients with atherosclerosis-thrombolysis in myocardial infarction 50 (TRA2 degrees P-TIMI 50). Circulation. 2016:133:997-1005.

22. Anand SS, Caron F, Eikelboom JW, et al. Major adverse limb events and mortality in patients with peripheral artery disease: the COMPASS trial. J Am Coll Cardiol. 2018;71:2306-15.

23. Gerhard-Herman MD, Gornik HL, Barrett C, et al. 2016 AHA/ACC guideline on the management of patients with lower extremity peripheral artery disease: a report of the American College of Cardiology/American Heart Association Task Force on Clinica Practice Guidelines. J Am Coll Cardiol. 2017;69:e71-126.

24. Antithrombotic Trialists' Collaboration. Collaborative meta-analysis of randomised trials of antiplatelet therapy for prevention of death, myocardial infarction, and stroke in high risk patients. BMJ. 2002;324:71-86.

25. Janzon L, Bergqvist D, Boberg J, et al. Prevention of myocardial infarction and stroke in patients with intermittent claudication; effects of ticlopidine. Results from STIMS, the Swedish Ticlopidine Multicentre Study. J Intern Med. 1990;227:301-8

26. CAPRIE Steering Committee. A randomised, blinded, trial of clopidogrel versus aspirin in patients at risk of ischaemic events (CAPRIE). Lancet 1996:348:1329-39.

27. ClinicalTrials.gov. A study comparing cardiovascular effects of ticagrelor and clopidogrel in patients with peripheral artery disease (EUCLID). ClinicalTrials.gov Identifier: NCT01732822. Available at: https://clinicaltrials.gov/ct2/show/NCT01732822 (accessed 12 July 2021).

28. Gutierrez JA, Mulder H, Jones WS, et al. Polyvascular disease and risk of major adverse cardiovascular events in peripheral artery disease: a secondary analysis of the EUCLID trial. JAMA Netw Open. 2018;1:e185239.

29. ClinicalTrials.gov. Clopidogrel for high atherothrombotic risk and ischemic stabilization, management and avoidance (CHARISMA). ClinicalTrials.gov Identifier: NCT00050817. Available at: www.clinicaltrials.gov/ct2/show/NCT00050817 (accessed 12 July 2021).

30. Bhatt DL, Flather MD, Hacke W, et al. Patients with prior myocardial infarction, stroke, or symptomatic periphera arterial disease in the CHARISMA trial. J Am Coll Cardiol. 2007;49:1982-8

31. Cacoub PP, Bhatt DL, Steg PG, et al. Patients with peripheral arterial disease in the CHARISMA trial. Eur Heart $J$. 2009;30:192-201 
32. ClinicalTrials.gov. Prevention of cardiovascular events (eg, death from heart or vascular disease, heart attack, or stroke) death fin with prior heart attack using ticagrelor compared placebo on a background of aspirin (PEGASUS). placebo on a background of aspirin (PEGASUS). https://clinicaltrials.gov/ct2/show/NCT01225562 (accessed 12 July 2021).

33. ClinicalTrials.gov. A study comparing cardiovascular effects of ticagrelor versus placebo in patients with type 2 diabetes mellitus (THEMIS). ClinicalTrials.gov Identifier: NCT01991795. Available at: www.clinicaltrials.gov/ct2/show/NCT01991795 (accessed 12 July 2021).

34. Steg PG, Bhatt DL, Simon T, et al. Ticagrelor in patients with stable coronary disease and diabetes. N Eng/ I Med. 2019;381:1309-20.

35. Belch JJ, Dormandy J, Committee CW, et al. Results of the randomized, placebo-controlled clopidogrel and acetylsalicylic acid in bypass surgery for peripheral arterial disease (CASPAR) trial. J Vasc Surg. 2010;52:825-33.

36. ClinicalTrials.gov. CASPAR: clopidogrel and acetyl salicylic acid in bypass surgery for peripheral arterial disease ClinicalTrials.gov Identifier: NCT00174759. Available at: https://clinicaltrials.gov/ct2/show/NCT00174759 (accessed 12 July 2021)

37. Qureshi MI, Li HL, Ambler GK, et al. Antiplatelet and anticoagulant use in randomised trials of patients undergoing endovascular intervention for peripheral arterial disease: systematic review and narrative synthesis. Eur I Vasc Endovasc Surg. 2020;60:77-87.

38. Tepe G, Bantleon R, Brechtel K, et al. Management of periphera arterial interventions with mono or dual antiplatelet therapythe MIRROR study: a randomised and double-blinded clinical trial. Eur Radiol. 2012;22:1998-2006.

39. Strobl FF, Brechtel K, Schmehl J, et al. Twelve-month results of a randomized trial comparing mono with dual antiplatelet of a randomized trial comparing mono with dual antiplatelet
therapy in endovascularly treated patients with peripheral therapy in endovascularly treated patients with periph

40. Aboyans V, Ricco JB, Bartelink MEL, et al. 2017 ESC guidelines on the diagnosis and treatment of peripheral arterial diseases, in collaboration with the European Society for Vascular Surgery (ESVS): document covering atherosclerotic disease of extracranial carotid and vertebral, mesenteric, renal, uppe and lower extremity arteries. Endorsed by: the European stroke Organization (ESO) the Task Force for the Diagnosis and Treatment of Peripheral Arterial Diseases of the European Society of Cardiology (ESC) and of the European Society for Vascular Surgery (ESVS). Eur Heart J. 2018:39:763-816.

41. Allemang MT, Rajani RR, Nelson PR, et al. Prescribing patterns of antiplatelet agents are highly variable after lower extremity of antiplatelet agents are highly variable after lower extremit
endovascular procedures. Ann Vasc Surg. 2013;27:62-7.

42. Laird JR, Katzen BT, Scheinert D, et al. Nitinol stent implantation versus balloon angioplasty for lesions in the superficial femora artery and proximal popliteal artery: twelve-month results from the RESILIENT randomized trial. Circ Cardiovasc Interv. 2010;3:267-76

43. Schillinger M, Sabeti S, Dick $P$, et al. Sustained benefit at 2 years of primary femoropopliteal stenting compared with balloon angioplasty with optional stenting. Circulation. 2007;115:2745-9.

44. Krankenberg H, Schluter M, Steinkamp HJ, et al. Nitinol stent implantation versus percutaneous transluminal angioplasty in superficial femoral artery lesions up to $10 \mathrm{~cm}$ in length: the femoral artery stenting trial (FAST). Circulation. 2007:116:285-92.

45. Hess CN, Norgren L, Ansel GM, et al. A structured review of antithrombotic therapy in peripheral artery disease with a focus on revascularization: a TASC (InterSociety Consensus for the Management of Peripheral Artery Disease) Initiative Circulation. 2017;135:2534-55.

46. Morrow DA, Braunwald E, Bonaca MP, et al. Vorapaxar in the secondary prevention of atherothrombotic events. N Eng J Med. 2012;366:1404-13

47. ClinicalTrials.gov. Trial to assess the effects of Vorapaxar (SCH 530348; MK-5348) in preventing heart attack and stroke in patients with atherosclerosis (TRA $2^{\circ} \mathrm{P}$ - TIMI 50) (P04737). ClinicalTrials gov Identifier: NCT00526474. Available at: www.clinicaltrials.gov/ct2/show/NCT00526474 (accessed 12 July 2021).

48. Efficacy of oral anticoagulants compared with aspirin after infrainguinal bypass surgery (The Dutch Bypass Oral Anticoagulants or Aspirin Study): a randomised trial. Lancet. 2000;355:346-51.

49. ClinicalTrials.gov. Warfarin and antiplatelet vascular evaluation ClinicalTrials.gov Identifier: NCT00125671. Available at: www.clinicaltrials.gov/ct2/show/NCT00125671 (accessed 12 July 2021).

促 Anand S, Yusuf S, et al. Oral anticoagulant and antiplatelet therapy and peripheral arterial disease. $N$ Eng/ I Med. 2007:357:217-27.

51. ClinicalTrials.gov. An efficacy and safety study for rivaroxaba in patients with acute coronarys. ClinicalTrials.gov Identifier: NCT00809965. Available at: https://clinicaltrials.gov/ct2/show/ NCT00809965 (accessed 12 July 2021).

52. Mega JL, Braunwald E, Wiviott SD, et al. Rivaroxaban in patients with a recent acute coronary syndrome. N Eng $\lrcorner$ Med. 2011;366:9-19

53. Eikelboom JW, Connolly SJ, Bosch J, et al. Rivaroxaban with or without aspirin in stable cardiovascular disease. N Engl J Med. 2017;377:1319-30

54. ClinicalTrials.gov. Rivaroxaban for the prevention of major cardiovascular events in coronary or peripheral artery disease (COMPASS). ClinicalTrials.gov Identifier: NCT01776424. Available at: https://clinicaltrials.gov/ct2/show/NCT01776424 (accessed 12 July 2021).

55. Anand SS, Bosch J, Eikelboom JW, et al. Rivaroxaban with or without aspirin in patients with stable peripheral or carotid artery disease: an international, randomised, double-blind placebo-controlled trial. Lancet. 2018;391:219-29.

56. Janssen Pharmaceuticals Inc. Xarelto (rivaroxaban) tablets, for oral use [package insert]. 2020. Available at: www.accessdata.fda.gov/drugsatfda_docs/ label/2020/202439s031,022406s035lbl.pdf (accessed 6 July 2021

57. Janssen Pharmaceuticals Inc. Xarelto (rivaroxaban) [summary of product characteristics]. 2020. Avallable at: www.ema.europa. eu/en/documents/product-information/rivaroxaban-accordepar-product-information_en.pdf (accessed 6 July 2021).

58. Bonaca MP, Bauersachs RM, Anand SS, et al. Rivaroxaban in peripheral artery disease after revascularization. N Eng I Med. 2020:382:1994-2004

59. ClinicalTrials.gov. Efficacy and safety of rivaroxaban in reducing the risk of major thrombotic vascular events in subjects with symptomatic peripheral artery disease undergoing periphera symptomatic peripheral artery disease undergoing periph (VOYAGER PAD). ClinicalTrials.gov Identifier. NCT02504216. (VOYAGER PAD). Clinicaltrials.gov Identifier. NCT02504216. Available at: https://clinicatr
(accessed 12 July 2021)

60. Hiatt WR, Bonaca MP, Patel MR, et al. Rivaroxaban and aspirin in peripheral artery disease lower extremity revascularization: impact of concomitant clopidogrel on efficacy and safety. Circulation. 2020;142:2219-30. 